\title{
Depth of processing and test anxiety in landscape recognition
}

\author{
DAVID J. MILLER, JOHN H. MUELLER, ALVIN G. GOLDSTEIN, \\ and TERRY L. POTTER \\ University of Missouri, Columbia, Missouri 65201
}

\begin{abstract}
Subjects selected on the basis of test anxiety scores made a judgment about landscape photographs during the study phase of a recognition experiment. The orienting tasks required attention to features that involved either deep (geographic location) or shallow (colorfulness) processing. Test performance was better for subjects who had made the deep decision during study. Compared to low-anxiety subjects, the criterion was higher for high-anxiety subjects following deep processing, but it was lower for high-anxiety subjects following shallow processing.
\end{abstract}

Craik and Lockhart (1972) have recently proposed that differences in verbal memory can be explained by differences in the depth of processing a stimulus word receives. At one level, the stimulus is processed primarily in terms of physical attributes (e.g., sound), while deeper levels of processing involve the semantic content of the word. According to this analysis, more durable memory traces are created by deeper processing. One procedure that has been used to manipulate the depth of processing requires the subject to perform some incidental task that orients attention to specific features, followed by an unannounced retention test (e.g., Hyde \& Jenkins, 1973). Retention is typically better when subjects perform a deep task (e.g., rating items for pleasantness) compared to a shallow task (e.g., detecting a particular letter). These results are consistent with the idea that a subject's activities during study will determine how well items are remembered, even without the intentional set to remember.

Most studies dealing with the depth analysis have utilized verbal materials. However, some recent studies have examined the effect of various processing tasks on facial recognition. It has been found that judgments about such traits as honesty and intelligence lead to better recognition than decisions such as gender and weight (e.g., Bower \& Karlin, 1974; Strnad \& Mueller, 1977; Winograd, 1976).

The present experiment explored the relationship setween depth of processing in the orienting task and ecognition performance for pictures of landscapes. The semantic-nonsemantic distinction is not as clear with such pictorial material as with words. However, $t$ is still possible to identify attributes that differentially Iffect retention (e.g., Mandler \& Ritchey, 1977). rresumably, then, the nature of the orienting task

Requests for reprints should be sent to John H. Mueller, 'sychology Department, 210 McAlester Hall, University of Aissouri, Columbia, Missouri 65201. would determine which information is processed, with corresponding effects on retention.

The other factor in this study was test anxiety. Research has indicated that high arousal states may orient attention to the physical characteristics of stimuli at the expense of semantic processing (cf. Eysenck, 1977). The interaction of this arousal-anxiety deficit in cue utilization with the orienting task procedure was of interest in this study. Previous research using photographs of faces (Mueller, Bailis, \& Goldstein, Note 1) compared high and low test-anxious subjects following deep or shallow orienting tasks. An unannounced recognition test revealed superior performance by low-anxiety subjects and by subjects who made the deeper judgments during study, with no interaction of anxiety and task. The present study examined these factors in landscape recognition.

\section{METHOD}

\section{Subjects and Design}

Forty subjects were selected from an introductory psychology course on the basis of test anxiety scores (Sarason, 1972). High-anxiety subjects had scores greater than 19 (mean $=25.9)$ and low-anxiety subjects had scores less than 15 (mean = 9.6). They were randomly assigned to the four groups formed by the factorial combination of test anxiety (high, low) and orienting task (shallow, deep), with five men and five women in each cell.

\section{Materials}

The stimuli were 60 color slides of varied naturalistic scenes (e.g., mountains, seashores, fields, streams, and waterfalls). Pictures with distinctive cues (e.g., people, buildings, telephone poles, bridges) were eliminated to increase homogeneity.

\section{Procedure}

Subjects were led to believe that the study phase of the experiment was concerned only with reaction times for various decisions. For the deep task, subjects compared pairs of pictures to decide which of the two scenes was in the United States. For the shallow orienting task, subjects selected the more colorful of the two slides. In addition to an intuitive difference in the depth of processing required by these tasks, these 
particular tasks were selected on the basis of pilot work comparing several tasks (Potter \& Goldstein, Note 2). ${ }^{1}$

Subjects were told to indicate their decisions as rapidly as possible, by pressing one of two buttons on a device at their desk, and then to continue watching the slides. Wires ran from the button box into another room, but neither response choice nor latency was actually recorded. The subjects were told to use whatever criteria seemed appropriate and helpful in making their decisions. The subsequent recognition test was not mentioned.

Subjects were run in small groups of up to eight per session. The 30 study slides were presented in pairs at a $5-\sec$ rate. Pairs were always of like content, for example, two waterfalls. Following the last pair of slides, there was a 3-min interval during which the experimenter conversed with the subjects. The recognition test was then administered. The 30 old slides were randomly mixed with 30 new distractor slides and presented one at a time at a 5-sec rate. There was at least one distractor slide of content similar to that for each pair in the study set. Subjects were told to mark on their answer sheet whether or not they thought each slide had been used in the previous phase of the experiment. They were not required to limit themselves to 30 "old" responses, and no mention was made of guessing, either to encourage it or to suggest penalties for guessing.

Subjects filled out two 20-item questionnaires assessing situational anxiety, one just prior to the experiment and the other just after. One was the State Anxiety Inventory (Spielberger, Gorsuch, \& Lushene, 1970) and the other was the Thayer Activation-Deactivation Adjective Checklist (Thayer, 1967), counterbalanced as first and second tests across subjects. Subjects selected for high and low test anxiety scores differed significantly $(\mathrm{ps}<.001)$ on both the state anxiety scores (means $=40.5$ vs. 35.5 ) and the Thayer scores (means $=46.1$ vs. 42.1). Situational anxiety differences would be expected, since most subjects perceive an experiment as an evaluation, and the general instructions encouraged this by stating that the experimenter was interested in the relationship between intelligence and the experimental task.

\section{RESULTS ${ }^{2}$}

The results of the recognition test are summarized in Table 1. The task proved quite difficult. The analysis of hit rates revealed that deep processing led to better performance than shallow processing $(F=20.40$, MSe $=.013$ ), but there was no main effect of anxiety nor any Task by Anxiety interaction (Fs $<1)$. The false alarm rate revealed a marginally significant tendency for high-anxiety subjects to make more misidentifications, primarily after shallow processing $(\mathrm{F}=3.20, \mathrm{MSe}=.015, \mathrm{p}<.08)$, but the task main effect and the Task by Anxiety interaction were not significant $(\mathrm{Fs}<2.10)$. Analysis of the total number of "old" responses (hits plus false alarms) revealed that more such responses were made after deep processing than after shallow $(\mathrm{F}=13.34, \mathrm{MSe}=27.438)$. The anxiety main effect and Task by Anxiety interaction were not significant $(\mathrm{Fs}<1.12)$.

Signal detection analyses were performed also, and the results are summarized in Table 1 . The $\mathrm{d}^{\prime}$ analysis revealed better performance following the deep task $(\mathrm{F}=5.54, \mathrm{MSe}=.210)$. (The shallow-high group's $\mathrm{d}^{\prime}$ was not significantly different from zero by a t test, although the other three groups were.) There was no anxiety main effect $(F=2.71)$ nor any Task by Anxiety interaction $(F=1.23)$. The analysis of the criterion measure $\beta$ revealed no task or anxiety main effects $($ Fs $<1)$. However, the Task by Anxiety interaction was significant $(\mathrm{F}=5.39, \mathrm{MSe}=.049)$, as high-anxiety subjects had the higher criterion given a deep task, while the low-anxiety subjects adopted the higher criterion following a shallow task. ${ }^{3}$

Sex of the subject was included as a factor in the preceding analyses. While there were no significant sex main effects, nor interactions of sex with either anxiety or task alone, there was a significant triple interaction of Sex by Task by Anxiety for false alarm rate $(F=17.41)$, total old responses $(F=14.24), d^{\prime}$ $(F=4.51)$, and $\beta(F=4.19)$, but not for hit rate $(F=1.52)$. The false alarm interaction indicated that high-anxiety males made more than low-anxiety males following deep processing, with no difference for shallow tasks, while high-anxiety females made fewer than low-anxiety females following deep processing, and high-anxiety females made more than low-anxiety females following shallow processing. In other words. deep processing led to fewer false alarms compared to shallow processing only for low-anxiety males and high-anxiety females. The $\mathrm{d}^{\prime}$ interaction indicated that deep processing was better than shallow except for low. anxiety females; while high anxiety generally hinderec performance, this was not true for females given the deep task. The $\beta$ interaction indicated that deer processing led to a higher criterion than shallow except for low-anxiety females; anxiety level seemec unimportant for males, but high anxiety lowerec

Table 1

Mean Recognition Performance By Orienting Task and Anxiety

\begin{tabular}{|c|c|c|c|c|c|c|c|c|c|c|c|c|}
\hline & \multicolumn{4}{|c|}{ All Subjects } & \multicolumn{4}{|c|}{ Males Only } & \multicolumn{4}{|c|}{ Females Only } \\
\hline & \multicolumn{2}{|c|}{ Deep } & \multicolumn{2}{|c|}{ Shallow } & \multicolumn{2}{|c|}{ Deep } & \multicolumn{2}{|c|}{ Shallow } & \multicolumn{2}{|c|}{ Deep } & \multicolumn{2}{|c|}{ Shallow } \\
\hline & High & Low & High & Low & High & Low & High & Low & High & Low & High & Low \\
\hline $\begin{array}{l}\text { Hit Rate } \\
\text { False Alarm Rate } \\
\text { Old Responses } \\
d^{\prime} \\
\beta\end{array}$ & $\begin{array}{r}.57 \\
.42 \\
29.70 \\
.40 \\
1.19\end{array}$ & $\begin{array}{r}.58 \\
.41 \\
29.70 \\
.48 \\
1.07\end{array}$ & $\begin{array}{r}.41 \\
.44 \\
25.40 \\
-.10 \\
.97\end{array}$ & $\begin{array}{r}.42 \\
.31 \\
21.90 \\
.30 \\
1.17\end{array}$ & $\begin{array}{r}.53 \\
.49 \\
30.80 \\
.13 \\
1.14\end{array}$ & $\begin{array}{r}.57 \\
.33 \\
27.00 \\
.63 \\
1.18\end{array}$ & $\begin{array}{r}.32 \\
.35 \\
20.00 \\
-.10 \\
.95\end{array}$ & $\begin{array}{r}.44 \\
.40 \\
25.20 \\
.11 \\
1.03\end{array}$ & $\begin{array}{r}.61 \\
.35 \\
28.60 \\
.68 \\
1.24\end{array}$ & $\begin{array}{r}.60 \\
.48 \\
32.40 \\
.33 \\
.95\end{array}$ & $\begin{array}{r}.49 \\
.53 \\
30.80 \\
-.10 \\
.98\end{array}$ & $\begin{array}{r}.39 \\
.23 \\
18.60 \\
.49 \\
1.31\end{array}$ \\
\hline
\end{tabular}


criterion for shallow-processing females while raising it for deep-processing females. The reason for these triple interactions is unclear, and they seem best interpreted cautiously, in view of the small number of subjects $(n=5)$ in the cells involved in the triple interaction.

\section{DISCUSSION}

This experiment found that a deep orienting task led to better recognition of landscape scenes than a shallow task. This extends the orienting task findings to pictorial material other than facial photographs. Furthermore, this difference was not accompanied by a general criterion shift for levels of processing, appearing instead to be a matter of differential sensitivity (hits and $\mathrm{d}^{\prime}$ ).

However, there was some indication of a criterion shift as a function of anxiety level, as revealed by the Task by Anxiety interaction for $\beta$. It appeared to be the case that, compared to low-anxiety subjects, high anxiety led to more cautious responding (higher $\beta$ ) in conjunction with a deep task, but less cautious responding after shallow processing. Examined more closely, though, this criterion shift was more evident for women than men (and this may be a factor in the other higher order interactions). One could speculate that this criterion shift is analogous to the observation (cf. Eysenck, 1977) that arousal focuses attention on the physical (nondominant) properties of words at the expense of deep (dominant) features. That is, shallow/high-anxiety subjects set a low criterion on the test since they had processed in a compatible way during incidental study, while deep/high-anxiety subjects set a high criterion on the test because they normally make test decisions using shallow features but had just processed deeply during incidental study. In any event, while anxiety did not yield strong main effects, its involvement in interactions suggests a role in pictorial recognition which warrants further examination.

\section{REFERENCE NOTES}

1. Mueller, J. H., Bailis, K. L., \& Goldstein, A. G. Depth of processing and anxiety in facial recognition. Paper read at Midwestern Psychological Association, Chicago, 1978.

2. Potter, T. L., \& Goldstein, A. G. Levels of processing in pictorial recognition memory. Unpublished manuscript, 1976.

\section{REFERENCES}

Bower, G. H., \& KARIIN, M. B. Depth of processing pictures of faces and recognition memory. Journal of Experimental Psychology, 1974, 103, 751-757.

CRAIK, F. I. M., \& Lockhart, R. S. Levels of processing: A framework for memory research. Journal of Verbal Learning and Verbal Behavior, 1972, 11, 671-684.
EysenCK, M. W. Human memory: Theory, research and individual differences. Oxford: Pergamon Press, 1977.

GRIER. J. B. Nonparametric indices for sensitivity and bias: Computing formulas. Psychological Bulletin, 1971, 75, 424-429.

HydE, T. S., \& Jenkins, J. J. Recall for words as a function of semantic, graphic, and syntactic orienting tasks. Journal of Verbal Learning and Verbal Behavior, 1973, 12, 471-480.

Johinson, N. S. A note on the use of $A^{\prime}$ as a measure of sensitivity. Journal of Experimental Child Psychology, 1976, 22, 530-531.

MANDleR, J. M., \& Ritchey, G. H. Long-term memory for pictures. Journal of Experimental Psychology: Human Learning and Memory, 1977, 3, 386-396.

SARAson, I. G. Experimental approaches to test anxiety: Attention and the uses of information. In C. D. Spielberger (Ed.), Anxiety: Current trends in theory and research (Vol. 2). New York: Academic Press, 1972.

SPielberger, C. D., Gorsuch, R. L., \& Lushene, R. E. Manual for the state-trait anxiety inventory. Palo Alto, Calif: Consulting Psychologists Press, 1970.

StrNad, B. R., \& Mueller, J. H. Levels of processing in facial recognition memory. Bulletin of the Psychonomic Society, 1977, 9, 17-18.

ThAYER, R. E. Measurement of activation through selfreport. Psychological Reports, 1967, 20, 663-678.

WINOGRAD, E. Recognition memory for faces following nine different judgments. Bulletin of the Psychonomic Society, $1976,8,419-421$.

\section{NOTES}

1. Several judges familiar with the levels analysis of verbal memory were asked to rate the depth of processing required for various orienting tasks designed for use with landscapes. Geographic location was ranked as the extreme example of deep processing, and color was ranked as an example of shallow processing, with some other features ranked at intermediate levels. A recognition test comparing many of these decisions showed that the location task led to a higher hit rate than the color task, with means of .71 and .53 , respectively $[F(3,30)=10.95, \mathrm{p}<.05]$.

2. All effects described as significant involve $p<.05$, unless otherwise noted. All Fs have 1 and 32 degrees of freedom.

3. Nonparametric analyses were also performed (Grier, 1971; Johnson, 1976). The main difference was that the $A^{\prime}$ analysis failed to reveal the significant task main effect that the $d^{\prime}$ analysis showed $(F=2.04)$. Otherwise, the conclusions were identical. 